\title{
An Exploration of Capitation Payments for Healthcare Services Using a Multi-agent Simulation
}

\author{
Fu-Ren Lin \\ Institute of Service Science of National Tsing Hua University \\ Hsinchu, Taiwan \\ frlin@mx.nthu.edu.tw \\ Rung-Wei Po \\ Institute of Technology Management of National Tsing Hua University, Taiwan \\ d9673802@oz.nthu.edu.tw \\ Min-Chen Lin \\ Institute of Service Science of National Tsing Hua University \\ augua1216@gmail.com \\ Wen-Ya Lin \\ Institute of Service Science of National Tsing Hua University \\ conanhaha@gmail.com \\ Benjamin Chang \\ Woodrow Wilson School of Public and International Affairs of Princeton University \\ Princeton, USA \\ bachang@princeton.edu
}

\begin{abstract}
The soaring cost of healthcare service demands alternative payment schemes to control expenditures while maintaining quality of service. The capitation payment scheme is a tentative substitute for the Fee-For-Service (FFS) scheme. A pilot capitation program study was conducted by the Bureau of National Health Insurance (BNHI) to evaluate the feasibility of a national rollout. Estimating the potential outcomes of a specific pilot program, the multi-agent simulation approach is used to model and simulate the service system under different settings. In this study, we chose the Zhishan Community Healthcare Group (CHG) service system as an example. The model was built and validated according to publicly available statistical and transactional data from Zhishan-CHG, and estimated the system's financial sustainability under different conditions. Five scenarios were explored in the experiment, simulating the capitation payment scheme over a period of three years. The results are summarized as follows: (1) medical cost expenditure per person can be reduced as more patients are enrolled in the capitation program; (2) the service model with a health promotion agent outperforms the model without one in an FFS cost control system; (3) higher quality and retention rate improve capitation income; (4) the financial risk of the CHG service system can be mitigated by excluding inpatient medical costs from the virtual points assigned by $\mathrm{BNHI}$, and (5) simulation results show a positive financial outcome for Zhishan-CHG. These simulation results could be potentially used by the BNHI to revise its policy in terms of the population for one $\mathrm{CHG}$ and the coverage of medical services of a capitation payment system, such that the financial risk of the CHG could be reduced and the capitation policy sustained.
\end{abstract}

Keywords: Capitation payment, healthcare services, multi-agent simulation, Swarm 


\section{Introduction}

Healthcare payment reform in Taiwan has become an important issue due to the average growth rate of National Health Insurance (NHI) expenditures beginning to exceed the growth rate of $\mathrm{NHI}$ premium incomes (Chan, 2010). An NHI deficit represents a large governmental financial burden, and in turn increases the encumbrance of taxpayers. In order to resolve the increasing gap in the $\mathrm{NHI}$ budget, the Department of Health $(\mathrm{DOH})$ proposed a 'second-generation NHI' policy to increase premium incomes, and launched a capitation pilot plan aimed at reducing medical service expenditures through incentives to promote health (BNHI, 2011). In the context of a capitation payment system, medical service providers could be motivated to enhance collaboration in order to better take care of their patients, which would reduce patients' medical expenditures and thus increase provider revenue.

On the one hand, the healthcare service system under $\mathrm{NHI}$ grants patients the freedom to access any medical services covered by $\mathrm{NHI}$ policy, which makes it difficult for individual medical service providers to manage patients' health. On the other hand, since Taiwan's national health policy is a single payer system, diagnosis and treatment information is centrally collected for reimbursement purposes, which enables the $\mathrm{NHI}$ to assess healthcare ecosystems in a timely manner (Nealon \& Moreno, 2003). Therefore, the up-to-date healthcare information provided by $\mathrm{NHI}$ is useful for planning and conducting capitation projects. Although mathematical models have been commonly used to generate system models, they typically examine only a small number of variables over a relatively short period of time, and thus fail to represent the systems as the diverse and dynamic enterprises that they are (Heirich, 1998). In particular, mathematical models usually ignore the effects of accumulation, time, resource constraints, and behavioral feedback. As such, the complex and diverse natures of health service systems make agent-based modeling the most suitable methodology for study. In order to evaluate the impact of interventions on the outcomes of health service systems, multi-agent simulation systems can be used to model and simulate interactions among stakeholders, as well as identify key factors for enhancing the service systems (Sterman, 2006).

In this study, we explored the value cocreated by the stakeholders of a community healthcare group ( $\mathrm{CHG}$ ) as a service system under the capitation payment scheme. In general, the annual sum of an enrolled patient's capitation payment is predetermined and assigned to the corresponding medical service provider regardless of whether the patient seeks care there. Zhishan-CHG is the only CHG-typed capitation model among the seven experimental capitation programs currently active in Taiwan. It promotes patients' health through an organizational collaboration with Zhishan Healthy Living Social Enterprise (HLSE), aiming to strengthen community relationships to encourage patient loyalty to the $\mathrm{CHG}$. In this study, we discuss the results of simulating different scenarios for the Zhishan-CHG service system, identifying important factors determining the sustainability of the capitation payment mechanism in a CHG-typed system. We conducted three experiments based on a multi-agent simulation platform called Swarm under different settings tuned by the number of patients, patient retention rate, and health quality index. These simulation results could be used by practitioners to revise their current capitation system. For the NHI, the results could be used to revise reimbursement policies under different service systems. For legislators, the results could be used to revise healthcare policy to enhance the sustainability of Taiwan's healthcare systems. The findings could also be referenced by other nations. 


\section{Literature review}

\section{Payment Reform and Capitation}

Payment schemes for healthcare services today reward volume rather than value. The most common way of paying for healthcare services today is the Fee-For-Service (FFS) system (Miller, 2009). This system, however, encourages over-testing, over-diagnosis and over-treatment, as providers get paid regardless of the quality or outcomes of care provided. This has specifically held true in Taiwan: the $\mathrm{NHI}$ has faced a rapid increase in healthcare costs following implementation of the policies of low-cost universal coverage and freedom of choice. The first $\mathrm{NHI}$ deficit occurred in 1998, resulting in the implementation of a global budget in 2002. In Taiwan, there are five sectoral global budgets: hospital expenditures, primary care, dental care, traditional medicine, and kidney dialysis (Cheng, 2009). This does not replace FFS, but rather is complementary, requiring hospitals to constrict fees according to precapped total amounts of reimbursement. This payment reform also moved the payment unit from the single medical service provider to a regional budget control. Meanwhile, there were ongoing adjustments in premium baselines, copayments, and resource usage reviews, including of the pricing of medicine.

However, this change did not solve the problem of over-consuming the healthcare budget due to competition among regional hospitals. In effect, a zero-sum game was created, as hospitals raced to claim a large share of the global budget before others, resulting in overruns of the global budget regardless of theoretical caps (Cheng, 2003). As a result, Taiwan in 2010 had the highest rate of outpatient visits in the world, averaged an increase of $69 \%$ in emergency visits as compared with 2009, and more generally experienced an expansion of the number of total operations performed to $150 \%$ of ten years prior (BNHI, 2011).

In 2011, the Bureau of $\mathrm{NHI}(\mathrm{BNHI})$ proposed a capitation payment model aimed at mending the financial gap by reducing the medical points claimed by service providers. "Capitation" payment models are designed to control the number of episodes of care as well as the cost of individual episodes. The basic concept is for a provider (or a group of providers, working in a coordinated fashion) to receive a single payment to cover all of the services that their enrolled patients need during a specific period of time, regardless of how many or few episodes of care the patients experience (Miller, 2009). In the USA, medical service providers generally contract with a health maintenance organization (HMO) known as an independent practice association (IPA) which enlists providers to care for HMO-enrolled patients. Remuneration is based on the average expected healthcare costs of that patient, with greater payment for patients with significant medical history. Rates are also affected by age, race, sex, type of employment, and geographic location, as these factors typically influence the cost of providing care. In England, there exists a capitation scheme organized by geography in which a gatekeeper mechanism with more than 100 health authorities is used. In Canada, there are 17 regional health authorities, also with a gatekeeper mechanism. Australia, Finland, Germany, New Zealand, Sweden and Norway have also adopted similar ways of implementing capitation (Rice \& Smith, 1999). In Taiwan, in contrast, the NHI has drawn up an open form of global capitation different from other countries. In particular, enrolled patients enjoy unfixed contractual rates, freedom of choice, and universal coverage, features which force providers to undertake uncertain risks when adopting the capitation scheme.

Generally, capitation models can be organized into four categories: per-memberper-month case management payments, practice capitation systems, partial global capitation systems, and global capitation systems (Cleveland, 2012). On one hand, healthcare policymakers believe that implementing global capitation will encourage providers to become more efficient due to the assumption of risk. On the 
other hand, providers may under-test, underdiagnose, and under-treat patients (Blackwell, Gutmann, \& Gutmann, 1988; Hillman, Pauly, \& Kerstein, 1989). In Taiwan, the NHI has proposed three types of healthcare capitation pilot plans. Participating medical institutes would submit their own health promotion and integrated medical care proposals based on a model chosen from three options: the region model, the loyalty model, and the CHG model. In this experiment, the general public still retains their ability to seek treatments from preferred hospitals or clinics. Capitation income is mainly decided by surplus savings derived by subtracting the baseline from the claimed amount, which is adjusted by an index of health quality. Thus, medical service providers still need to spend effort on promoting health in order to retain enrolled patients. If patients' medical expenses decrease, providers accrue increased capitation incomes.

It is necessary to consider risk in a discussion of this model. Roberge and coauthors (2001) used the focus group technique to set up six groups from patients and physicians practicing in Montreal. Their results show that patients have relatively congruent visions of the notion of loyalty when they are clearly associated with a particular physician. Their findings also indicate that systematic risks inherent to this model include loyalty and personal behavior changes in the enrolled patients. To share this systematic risk, the $\mathrm{NHI}$ decided to absorb $50 \%$ of any capitation losses suffered by participating medical institutions.

Many healthcare payment systems have been tried, such as FFS, global budget, pay for performance, DRG, and capitation. Although each has its strengths and weaknesses, the common goal is to enhance the health condition of the enrolled patients under certain budget constraints. Moreover, raising sufficient funds for health care is imperative, but just having money will not, in itself, ensure universal coverage (WHO, 2010). In other words, payment reform alone will not transform the healthcare delivery of medical institutes, but healthcare reform which combines financial incentives with organizational and cultural changes could lead to better healthcare service (Kahn, 2009).

\section{Value Co-creation and Community Participation in Health Promotion}

In the healthcare system, the value of enabling people to share responsibility for managing their own health with their healthcare service providers is widely recognized. In the value co-creation model, value is determined "in use" through the activities and interactions of customers with their service providers and others (Lusch, Vargo, \& O'Brien, 2007; Vargo \& Lusch, 2008). Patient compliance represents a willingness to co-create value with medical professionals. Basic compliance includes following the instructions of a healthcare service provider, such as visiting the clinic as directed, following an instruction to take medicine, or keeping a journal of their daily activities. Such compliance behaviors have been shown to result in improved self-reports of the individual's health status, perceptions of goal attainment, and satisfaction with health services (Fattal, Lampe, Barcelona, \& Muzina, 2005).

The value of healthcare services relies on a mutual exchange of benefits between patients and healthcare providers (Vargo, Maglio, \& Akaka, 2008). The core value is "health" co-created mutually. For example, from the perspective of a diabetic patient, $\mathrm{s} /$ he targets the goal of health, and might join a preventive group organized by a hospital to interact with his/her peers and medical professionals. From the perspective of a hospital, proactively engaging with patients can enhance the effects of therapy through the patients' social groups. McColl-Kennedy, Vargo, Dagger, Sweeney, and van Kasteren (2012) identified five roles, eight activities, and various corresponding interactions that underlie value co-creation in healthcare. 
McLeroy, Bibeau, Steckler, and Glanz (1988) proposed that an individual's behavior is shaped by a dynamic interaction with the social environment, including influences at the interpersonal, organizational, community, and policy levels. As such, a community group is widely used in many settings for managing health promotion activities, as well as for facilitating health empowerment and the development of skills (Hubley \& Copeman, 2008). A lack of group attachments is linked to a variety of negative effects on health, adjustment, and well-being (Baumeister \& Leary, 1995). It is well recognized that community groups can help individuals fill their need for a sense of belonging, peer cognition, and identification. Neuwelt (2012) regarded relationshipbuilding as a complex process occurring among different stakeholder groups, involving trust-building and information sharing between communities and health professions. The relationship enables people to feel comfortable to seek care, and professionals to mold services to meet patients' needs.

A customer service network can be conceptualized as a coalition forged by service providers (clinics, hospitals, or care centers), community groups, and individuals in healthcare. There are three types of coalitions, divided according to their organizational structure: organization-set coalitions, network coalitions and action-set coalitions (Roberts-DeGennaro, 1987). In our study, five primary care clinics united to form Zhishan-CHG as a network coalition to conduct a capitation payment pilot program in addition to their existing FFS services. Aiming to sustain the capitation program, Zhishan-CHG founded a healthcare life social enterprise (HLSE), a type of "organization-set coalition," and intended to leverage Zhishan-CHG and HLSE to promote healthy behavior and improved quality of life among residents through community participation. Achieving the vision of co-creating value for involved stakeholders is dependent on the many actors that make up the current Zhishan-CHG service system.

\section{Multi-agent Systems and the Swarm Simulation Platform}

Wooldridge and Jennings (1995) define an agent as a computer system situated in a certain environment. Such an agent is capable of autonomous action in this environment in order to meet its designated objectives. In an intelligent agent setting, three capabilities have to be included in the system design: reactivity, proactiveness, and social ability (Xiang, 2002). A multi-agent system can be defined as a collection of autonomous agents which coordinate activities in order to collectively solve problems that cannot be tackled by an agent individually (Nealon \& Moreno, 2003).

An important feature of multi-agent systems is that agents in such systems are autonomous (van der Hoek \& Wooldridge, 2008). This autonomy is reflected in the interrelation of agents and their human principals, as well as between agents. Due to the large number of agents in a multi-agent system, it is impractical for a human principal to guide each agent closely during its activities. For instance, there are far too many variables and unknown quantities in healthcare service systems to do anything except predict very broad trends across short timeframes, and even then the process is prone to embedded errors. This situation will remain for the foreseeable future.

The previous research on multi-agent systems provides a novel tool for simulating societies, shedding light on various kinds of social processes. Nealon and Moreno (2003) point out that many different kinds of problems in the healthcare domain, such as patient scheduling, organ and tissue transplant management, community care, information access, decision support systems, training, internal hospital tasks, and senior citizen care, have already been dealt with using intelligent agents. Moreno (2003) reported on the AgentCities.NET European project, which involved the construction of a worldwide network of agent-based platforms, called AgentCities, by a number of working 
groups (Moreno, Isern, \& Sánchez, 2003; Nealon, 2013).

We can conceptualize a healthcare service system as a multi-agent system in the view of systems design. From the perspective of systems design, the most important task is to develop mechanisms which enable agents to interact (e.g., cooperate, coordinate, negotiate) with other agents and carry out tasks delegated to them, particularly when agents cannot be assumed to share the same interests or goals. In our agent-based modeling, we intend to identify the benefits of agent-based healthcare systems for patients, medical service providers, health promoters, and government.

\section{Methodology}

Based on the prior research on multi-agent systems (Wooldridge \& Jennings,1995; Nealon \& Moreno, 2003), a multi-agent simulation methodology encoding the characteristics and behavior of an agent by the attributes and methods of an object with object-oriented programming languages has been widely used to model and evaluate the processes and outcomes of a multi-agent system. In this study, we adopted the multiagent simulation methodology to model and evaluate a capitation payment scheme by taking real data from Zhishan-CHG and $\mathrm{BNHI}$ as experimental settings. In this study, we used Swarm, a multi-agent simulation platform, for modeling and simulating healthcare service systems. Swarm is a general-purpose simulator based on an object-oriented framework for simulating concurrent, distributed artificial worlds (Hiebeler, 1994). The agents themselves are implemented as objects. Agents are created by taking a class from the Swarm libraries specialized in particular modeling domains, and then by instantiating one object per agent. Swarm uses the individual-based modeling approach, which allows an agent to have its internal state variables determine its actions (Hiebeler, 1994). The combination of individual actions determines the collective behavior of the whole group. Users can model the multi-agent system using built libraries with limited programming effort, which grants flexibility in modeling various system dynamics (Minar, Burkhart, Langton, \& Askenazi, 1996).

In the Swarm system, an agent itself can be a swarm of agents. For example, Swarm $A$ is composed of a set of agents, $\left(a_{1}, a_{2}, \ldots, a_{n}\right)$. An agent, e.g., $a_{k}$, itself is a swarm of agents called Swarm $k=\left(k_{1}, k_{2}, \ldots, k_{m}\right)$, where agent $a_{k}$ is the parent agent of agents in Swarm $k$. When child agents (e.g., agents in Swarm $k$ ) receive messages from their parent swarm's scheduler (e.g., Swarm A's scheduler), the behavior of that agent (e.g., $a_{k}$ ) is the result of actions performed by the swarm of constituent child agents (e.g., agents in Swarm k). This hierarchical inheritance, which can possess a depth of several layers, is called the nested inherent hierarchy property (Lin \& Pai, 2000; Lin, Tan, \& Shaw, 1999; Lin, Sung, \& Lo, 2003).

\section{Zhishan Community Healthcare Service System}

Zhishan clinic, which represents the Zhishan$\mathrm{CHG}$, is an integration of six primary care clinics, and is located in Taipei City's Shilin District as shown in Figure 1. The ZhishanCHG set the goal of creating better health for enrolled patients, and not the goal of more treatments (Yu, 2007). This principle guided their emphasis on prevention, early detection, correct diagnosis, and the avoidance of complications and measurements.

Figure 2 displays the community healthcare service system under the FFS scheme. People who have enrolled in $\mathrm{NHI}$ can access CHG services, although co-pays are required. The BNHI can redeem costs by evaluating the requests for reimbursement from the CHG. 


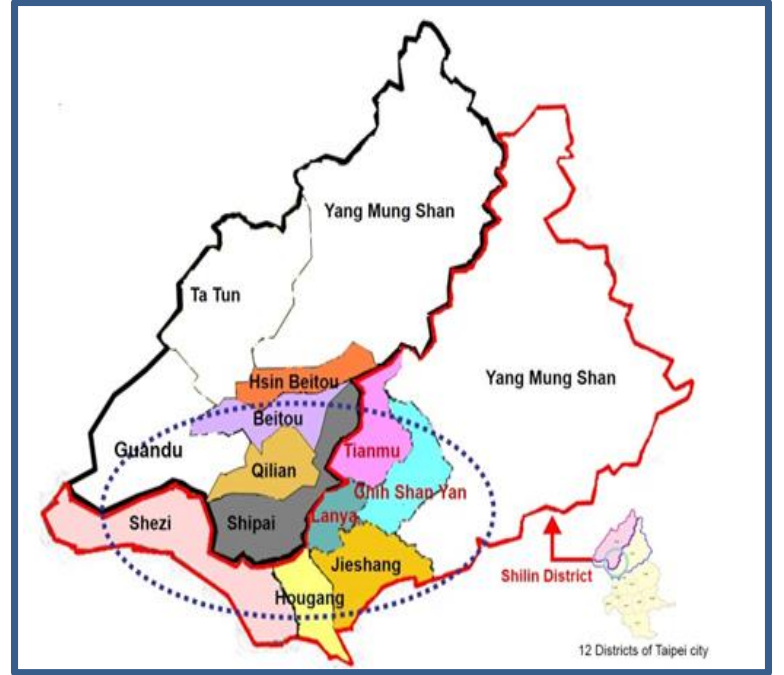

Figure 1 - Eight care neighborhoods of Shilin District in Taipei

Figure 3 illustrates the community healthcare service system under the capitation scheme. There are four major agents in this service system: policymaker (BNHI), provider (Zhishan-CHG), health promoter (HLSE), and customer (enrolled patient). The Zhishan-CHG provided medical services redeemed both by FFS reimbursement and by the surplus (or loss) of capitation payment, plus a certain additional amount from patient copayment. If Zhishan-CHG enhances patients' health status, on the one hand, the income from FFS and copayment will

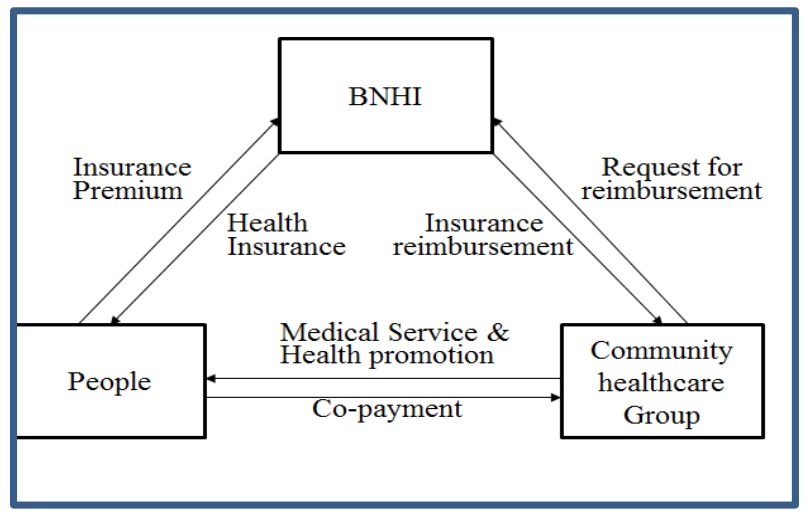

Figure 2 - The community healthcare service model in the FFS scheme decrease since the number of visits of enrolled patients will also decrease; on the other hand, it will obtain increased reward from the capitation payment. Zhishan-HLSE was founded in 2010 to provide health education, nutritional counseling, and circuit exercise programs, among other elements.

Functioning as a non-profit social enterprise, Zhishan-HLSE reinvested its profits into the program and was mostly staffed by volunteers. The HLSE was designed to play the role of a health promoter attempting to improve the health status of the enrolled patients of the Zhishan-CHG, so that the patients might reduce their usage of medical services and subsequently increase ZhishanCHG's rewards from capitation payments. In order to attract patients to enroll in the HLSEfacilitated programs as active members, patients were rewarded through being allowed to share in the surplus Zhishan-CHG capitation payment, which they could then spend on HLSE services. This service system hoped to create a virtuous cycle through mutual benefits for clinics and patients with the social enterprise, i.e., HLSE.

\section{Model Logic and Assumptions}

The model simulated changes over a threeyear period for all enrolled members of the Zhishan-CHG capitation system. The patients were categorized into 36 groups by gender and age. Furthermore, combining chronic and disease conditions, we created 6 state metrics: (1) healthy, (2) minor ailment, (3) severe illness, (4) chronic disease, (5) chronic with minor ailment for outpatient, and (6) chronic with severe illness. According to a causal model of the effects of subscription to HLSE services, each person was encoded with various health index values based on the frequency of attendance of HLSE activities. Enrollment in HLSE services makes people healthier, and results in less use of medical services. In this study, we 
emphasize the improvement of health conditions due to enrollment in HLSE services; we exclude the causes of injuries due to some accidents.
The other factor that affects capitation payment outcomes is patient loyalty, reflecting the extent to which medical service providers (internal/external $\mathrm{CHG}$ ) are preferred by patients.

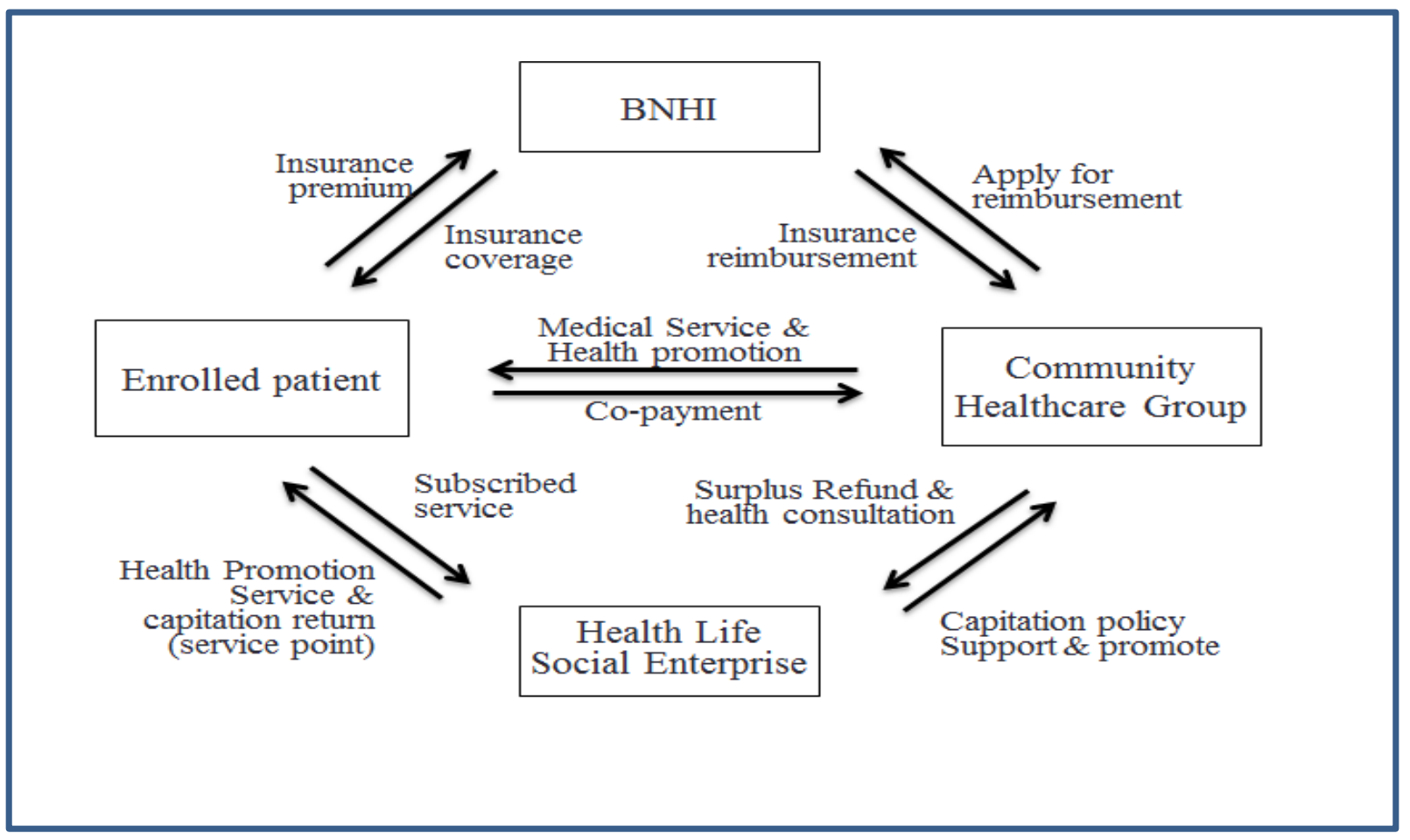

Figure 3 - The community healthcare service model in the capitation payment

The model captures all reported capitation costs associated with personal health promotion. The model compares payments resulting from the FFS and capitation models as claimed for outpatient treatments and inpatient treatments in hospitals. As the expenditure of medical services tends to increase under the FFS payment scheme due to the opportunity for increased payments from insurers, a patient able to maintain good health via preventive care and health-promoting activities would also have reduced medical expenditures under said FFS payment system. The capitation payment scheme will implicitly compensate the efforts made by medical institutes in promoting healthy behaviors and conducting preventive cares. Moreover, it may reduce volume-based competition among medical service providers.

\section{Capitation Service System Modeling and Simulation in Swarm}

We used Swarm 2.2, published by SDG in 2005 , to conduct simulations for five experiments. Swarm is a development tool for simulating the multi-agent system within a dynamic environment, and provides a framework to manage agents and the environment, as well as analytic tools for interactive experimentation or automated batch jobs (Hiebeler, 1994).

Figure 4 illustrates the characteristics of the nested inherent hierarchy of a multi-agent simulation system to model the healthcare service system for Zhishan-CHG. We model four types of agents: BNHI, CHG, enrolled patients, and HLSE. In the Swarm simulation platform, there are three built-in swarms, the 
observe, model, and statistics swarms, which facilitate the simulation process; in particular, the HSS Observe Swarm displays the simulation results, the Model Swarm is used to manage the interactions among simulated agents, and the Statistic Swarm provides basic statistical methods adopted by the Model Swarm to analyze the simulated systems. Figure 5 specifies the attributes and methods used in simulating capitation service systems on the Swarm simulation platform. The cost data of 5976 enrolled patients of Zhishan-CHG from late 2010 to early 2012 were used to calibrate the model's input variables and to confirm that its output faithfully reproduced historical metrics. Table 1 defines attributes and value ranges of each agent used for the simulation. In order to make the simulated system as valid as possible, we assigned values to attributes according to $\mathrm{NHI}$ statistics and clinical data from Zhishan-CHG.
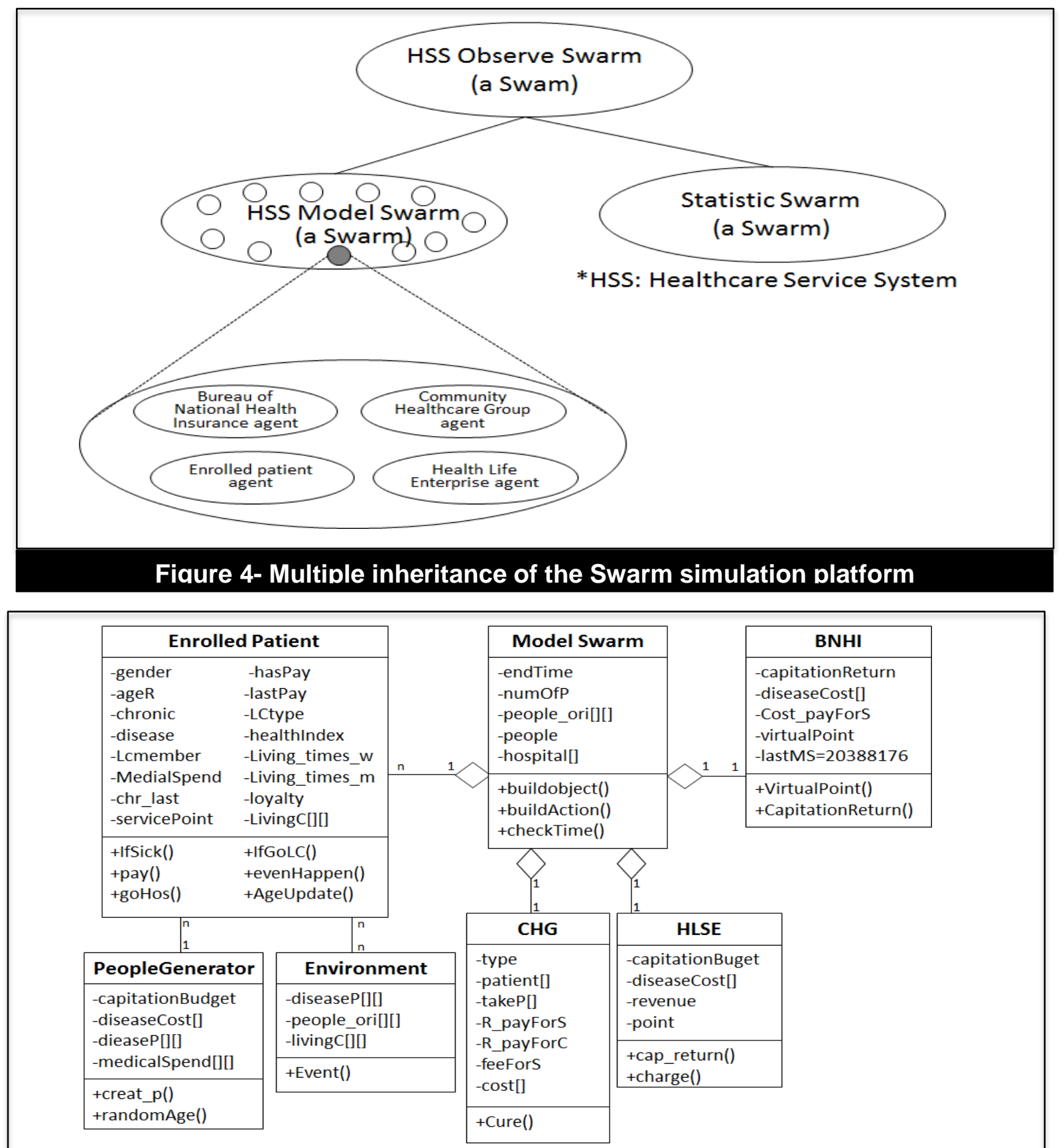


\begin{tabular}{|c|c|c|}
\hline Attribute & Value range & Definition \\
\hline \multicolumn{3}{|c|}{ Agent: Enrolled patient (Customer) } \\
\hline Gender & $\begin{array}{l}\text { 0: } \text { man } \\
\text { 1: women }\end{array}$ & Gender of enrolled patient \\
\hline Age & $\begin{array}{l}0: 0 \sim 4,1: 5 \sim 9,2: 10 \sim 14,3: \\
\text { 15 19, 4: 20 24, 5: 25 29, 6: } \\
\text { 30 34, 7: 35 39, 8: 40 44, 9: } \\
\text { 45 49, 10: 50 54, 11: 55 59, 12: } \\
\text { 60 64, 13: 65 69, 14: 70 74, 15: } \\
\text { 75 79, 16: 80 84, 17: 85up }\end{array}$ & Patient's age segmentation \\
\hline Chronic & 0: healthy; 1 : chronic & The chronic condition of an enrolled patient. \\
\hline Disease & $\begin{array}{l}\text { 0: health; } 1 \text { : minor ailment; } \\
\text { 2: severe illness; } 3 \text {. chronic } \\
\text { disease; } 4 \text { : chronic with minor } \\
\text { ailment for outpatient; } 5 \text { : chronic } \\
\text { with severe illness. }\end{array}$ & The health condition of an enrolled patient. \\
\hline HLSEMember & $\begin{array}{l}\text { 0: nonsubscriber } \\
\text { 1: subscriber }\end{array}$ & Patient became a subscriber of HLSE or not. \\
\hline EngagementType & $\begin{array}{l}\text { 0: subscribed but never joined. } \\
\text { 1: } 3 \text { to } 5 \text { times per week } \\
\text { 2: } 1 \text { to } 2 \text { times per week } \\
\text { 3: } 1 \text { to } 2 \text { times per month } \\
\end{array}$ & The frequency of a subscriber going to HLSE. \\
\hline HealthIndex & $\begin{array}{l}\text { Lctype }=1, \text { then healthIndex }=0.1 \\
\text { Lctype }=2 \text {, then healthIndex }=0.08\end{array}$ & $\begin{array}{l}\text { Based on the frequency of a subscriber to go to HLSE, it } \\
\text { will produce different values of healthlndex. The higher } \\
\text { value of healthlndex, the lower propability of illness. }\end{array}$ \\
\hline MedicalSpend & default $=0$ & $\begin{array}{l}\text { Patient's medical expenditure that will be accumulated in } \\
\text { a year. }\end{array}$ \\
\hline Loyalty & $\begin{array}{l}\text { Random produced 5-10 (round } \\
\text { number) }\end{array}$ & $\begin{array}{l}\text { The number will judge a patient whether going to a clinic } \\
\text { in the internal community health care group or an } \\
\text { external medical service provider. }\end{array}$ \\
\hline ServicePoint & default $=0$ & $\begin{array}{l}\text { HLSE's service points which can be redeemed in HLSE } \\
\text { services. (each point cost NT } \$ 10 \text { dollars) }\end{array}$ \\
\hline \multicolumn{3}{|c|}{ Agent: Community health care group (Providers) } \\
\hline Type & $\begin{array}{l}\text { 0: clinic } \\
\text { 1: hospital }\end{array}$ & The type of medical service provider. \\
\hline FeeForService & default $=0$ & $\begin{array}{l}\text { The medical expenditure consumed in a service provider } \\
\text { (e.g., hospital or clinic) that will be accumulated in a year. }\end{array}$ \\
\hline Cost & $\begin{array}{l}\text { 1: outpatient }=899 \\
\text { 2: inpatient }=17,439\end{array}$ & $\begin{array}{l}\text { The average medical payment of baseline-year reported } \\
\text { by Zhishan-CHG }\end{array}$ \\
\hline \multicolumn{3}{|c|}{ Agent: NHI (Policy maker) } \\
\hline CapitationReturn & $\begin{array}{l}\text { The surplus or loss of capitation } \\
\text { return. }\end{array}$ & $\begin{array}{l}\text { Virtual general medical payment minus actual medical } \\
\text { payment used will get the final return. }\end{array}$ \\
\hline VirtualPoint & $\begin{array}{l}\text { Type1: calculated by capitation } \\
\text { rule, all cost included. } \\
\text { Type2: calculated by capitation } \\
\text { rule, outpatient cost excluded. }\end{array}$ & $\begin{array}{l}\text { The base period of virtual global medical payment is one } \\
\text { year from the second half of } 2010 \text { to the first half of } 2011 \text {, } \\
\text { and then keep unchanged through three years of the } \\
\text { capitation project. }\end{array}$ \\
\hline MedicalGrow & $\begin{array}{l}0.00829 \text { (used in calculating } \\
\text { virtual general medical payment) }\end{array}$ & $\begin{array}{l}\text { Annual growth rate of the western medicine healthcare } \\
\text { providers and hospitals according to NHI statistics. }\end{array}$ \\
\hline GrowRate & $\begin{array}{l}0.03168 \text { (used in calculating } \\
\text { virtual general medical payment) }\end{array}$ & $\begin{array}{l}\text { Annual growth rate of the medical cost of each insured } \\
\text { according to NHI statistics. }\end{array}$ \\
\hline QualityIndex & $\begin{array}{l}0.85 \text { (used in calculating } \\
\text { capitation return or risk premium.) }\end{array}$ & $\begin{array}{l}\text { Medical quality index of the service provider according to } \\
\mathrm{NHI} \text { statistics. }\end{array}$ \\
\hline \multicolumn{3}{|c|}{ Agent: HLSE (health promoter) } \\
\hline Revenue & default $=0$ & $\begin{array}{l}\text { HLSE income coming from subscribers by cash or by } \\
\text { redeeming reward points. }\end{array}$ \\
\hline EndTime & The days of simulation & The time period of simulation set for the experiment. \\
\hline NumOfPatient & 5976 & The total enrolled patients of Zhishan-CHG \\
\hline Hospital & hospital[0]:Zhishan clinic & The type of a medical service provider. \\
\hline
\end{tabular}

Pacific Asia Journal of the Association for Information Systems Vol. 6 No. 2, pp.1-19 / June 2014 


\begin{tabular}{|l|l|l|}
\hline & $\begin{array}{l}\text { hospital[1]:External hospital } \\
\text { hospital[2]:External clinic }\end{array}$ & \\
\hline DiseaseProbability & $\begin{array}{l}\text { The probabilities of illness by } \\
\text { gender and age. }\end{array}$ & $\begin{array}{l}\text { The data comes from NHI annual report statistics of } \\
\text { inpatient and outpatient rates. }\end{array}$ \\
\hline EnrolledPeople & $\begin{array}{l}\text { De-personalized information of } \\
\text { enrolled patient of Zhishan clinic. } \\
\text { (e.g., age, gender, chronic } \\
\text { disease, medical cost) }\end{array}$ & The data provided by Zhishan clinic. \\
\hline
\end{tabular}

\section{Bureau of NHI}

$\mathrm{BNHI}$ is the policymaker agent and dominates all medical resources in this system. As a single payer, BNHI makes the rules of the game, as it pays for both FFS and capitation. If the number of virtual points claimed by the medical institute is smaller than its annual capitation points, the medical institute can be rewarded based on the surplus of capitation points; otherwise, the provider takes on the financial risk. Here, the virtual points denote the gross amount of units counted as points to specify the quantity of a medical service. As such, the business rules adopted in the simulation were specified as follows:

- Payment for FFS = the claimed medical points $\times$ point value (medical points are obtained based on medical services received by enrolled patients, and point value is a deducted value paid for medical services conducted by different medical institutes, such as hospital or clinics), where deducted value is set by $\mathrm{BNHI}$ based on the gross budget.

- Total virtual points $=$ baseline $\times(1.0+$ annual cost growth rate per person + western medicine growth rate) $\times$ number of enrolled patients; where the baseline is given based on the average medical points a person used in past year, and western medicine growth rate is given by the BNHI.

- Capitation points = the fixed total virtual points - annual total medical points claimed.
- Payment for capitation = basic rewards + medical quality rewards.

- Basic rewards $=$ Capitation points $\times 0.6 \times$ 1.0

- Medical quality rewards $=$ Capitation points $\times 0.4 \times \Sigma$ quality index $\times 1.0$

- Financial risk $=$ (negative capitation points) $\times(1-\Sigma$ quality index $) \times 0.5 \times 1.0$

\section{Community Healthcare Group (CHG)}

$\mathrm{CHG}$ is the agent representing the medical service provider, consisting of six primary clinics providing outpatient services. The expenditures of enrolled patients on visiting medical service providers not aligned with Zhishan-CHG are also counted, and consume capitation points. Thus, the CHG assumes the risk that enrolled patients might visit medical service providers not in the CHG. CHG revenue comes from $\mathrm{NHI}$ payments for FFS and capitation. Thus, a CHG seeks to enhance patient loyalty in order to increase their stickiness in terms of seeking healthcare treatment from $\mathrm{CGH}$. $\mathrm{CHG}$ agents are modeled by the following attributes.

- Providers include clinics within the $\mathrm{CHG}$, and clinics or hospitals not in the CHG, which have different medical costs.

- Medical cost includes the outpatient, inpatient, and average costs of ZhishanCHG calculated by medical points based on the baseline year.

- Returned rewards. Annual capitation income is shared with HLSE and enrolled 
patients in a contracted ratio when the CHG gets the surplus from capitation. HLSE shares $10 \%$ of the total capitation surplus and each enrolled patient shares $50 \%$ of his/her personal surplus.

\section{Enrolled patient}

This agent represents the customer enrolled in the capitation system. By $\mathrm{NHI}$ rule, in this experimental project, patients enrolled in the capitation payment system are not informed of their participation, and are granted the right to choose their healthcare providers. Half of the enrolled patents were assigned by $\mathrm{NHI}$, and the rest of them were chosen by Zhishan-CHG from their patient lists. To model patients' probabilities of getting sick and accessing HLSE programs, we define the probabilities as follows.

- Illness probabilities. The probability of a patient getting sick and visiting a medical institute was calculated by the number of visits of enrolled patients of Zhishan-CHG divided by the number of the enrolled patients multiplied by 365 based on the statistical data in the baseline year. The probability is calculated under different age ranges, genders, and types of medical services to generate 72 entries of probability.

- The choice of provider in specific conditions of illness. A patient has three options in choosing medical institutes when $\mathrm{s} /$ he is sick. S/he might be an outpatient treated in clinics within the $\mathrm{CHG}$ or clinics and hospitals not in the $\mathrm{CHG}$, or might be an inpatient hospitalized not in the CHG. S/he could also have chronic disease(s) and be prescribed medicine and asked to return regularly (in general, monthly).

- The frequency of attendance at HLSE services. Enrolled patients will be randomly assigned to subscribe to HLSE services. The frequency with which they attend HLSE services positively correlates with individual health status. The health index is used to specify a person's health status. In this study, we empirically collected the frequency with which HLSE members used its services.

\section{Health Life Social Enterprise (HLSE)}

This agent represents a health promoter. Zhishan-CHG founded HLSE to provide health enhancement activities to enrolled patients and community residents. Besides the subsidy from $10 \%$ of the surplus of the Zhishan-CHG capitation reward, the income of HLSE mainly depends on charging for service offerings and the annual membership fees of subscribers.

Based on the business' rules and the defined interactions among agents, we built the schedule of activities performed as actions by corresponding agents in a specified time frame by invoking functions embedded in the Swarm platform. The outputs of the simulated system were stored and then used for analysis in different circumstances set as input variables. In order to generate reliable results, the simulated service system was tested repetitively with different seeds obtained through random number generation to evaluate the probability of the occurrence of events.

\section{Simulation Results}

Before we evaluated the outputs of the simulated service system, we validated the accuracy of the simulation model as a good approximation of the real world system. We built the simulation system by taking 5976 enrolled patients of Zhishan-CHG as target customers according to the real transaction data. The probabilities of illness and the 




propensity of choosing one of the three optional medical institutes were set based on $\mathrm{NHI}$ statistical data regarding the enrolled patients of the CHG. The outputs of the simulation system were compared with the real world data in medical points in the first year of adopting the capitation payment scheme in order to validate the simulated system. The results show that the error rate is $4.48 \%$ in outpatient service and $0.88 \%$ in inpatient service, and that the average error rate is $3.09 \%$. These results indicate that the multi-agent simulation model can precisely simulate the real world system.

In order to evaluate the performance of the simulated service systems, we built the benchmarked simulation systems by setting the number of enrolled patients in the $\mathrm{CHG}$ to 5976. According to interviews with physicians of clinics, the general quality index of the CHG was reported at the level of 0.85 , the patient retention rate was set to $50 \%$, and the business rules adopted were set by BNHI.

\section{The Size of Enrolled Patients}

We wanted to know the financial outcome for Zhishan-CHG if the number of enrolled patients of the CHG was increased from 854 to 5976 . We calculated the average medical costs per person under four sizes of enrolled patients: $854,1500,3000$ and 5976. The results shown in Figure 6 indicate that the medical cost expended per person is reduced as the number of enrolled patients is increased.

\section{The Addition of HLSE to the Service System}

The traditional healthcare service system providing universal coverage is composed of three agents: insurers (BNHI), healthcare service providers, and patients as shown in Figure 2. FFS payment schemes often financially penalize providers for keeping people healthy, reducing errors and complications, and avoiding unnecessary care (Miller, 2009). The stakeholders of the Zhishan-CHG, in addition to these three agents, include the HLSE, a new agent to promote health, forming a service system with four types of agents as shown in Figure 3. 


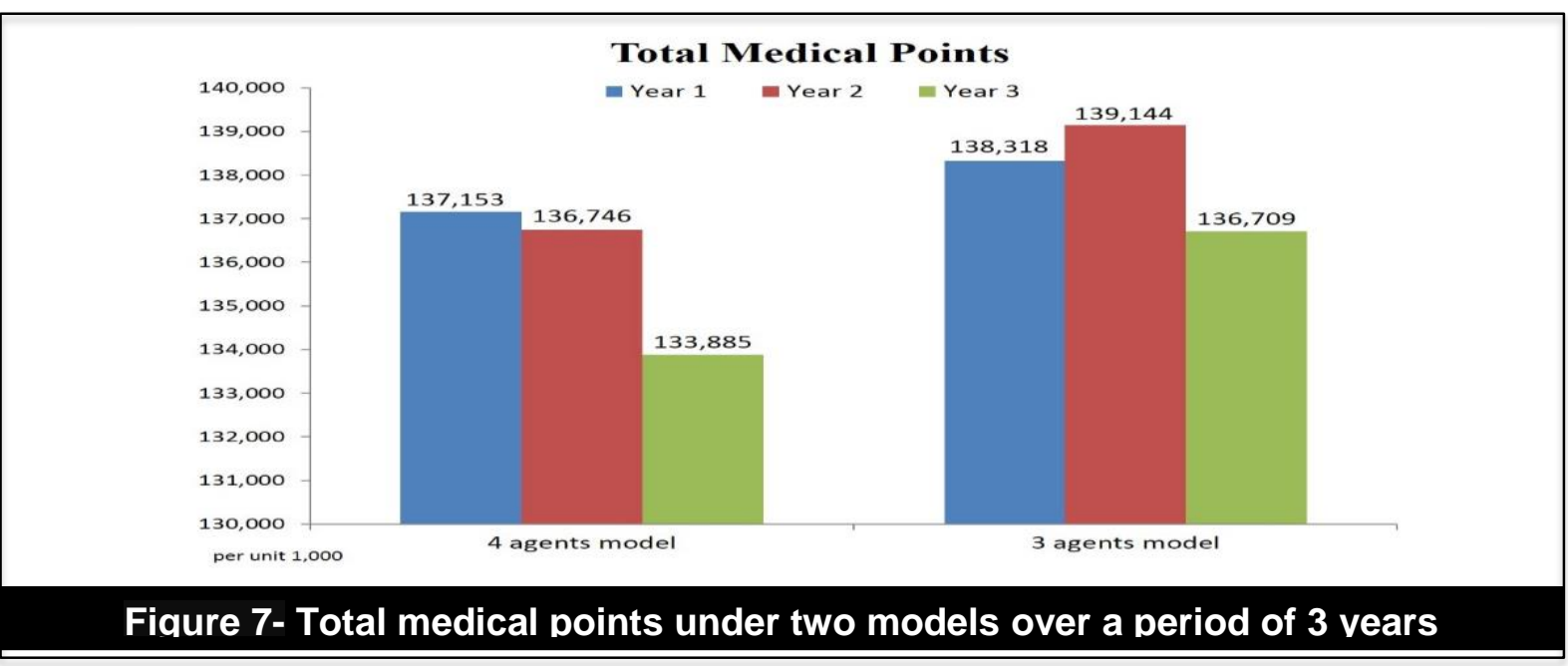

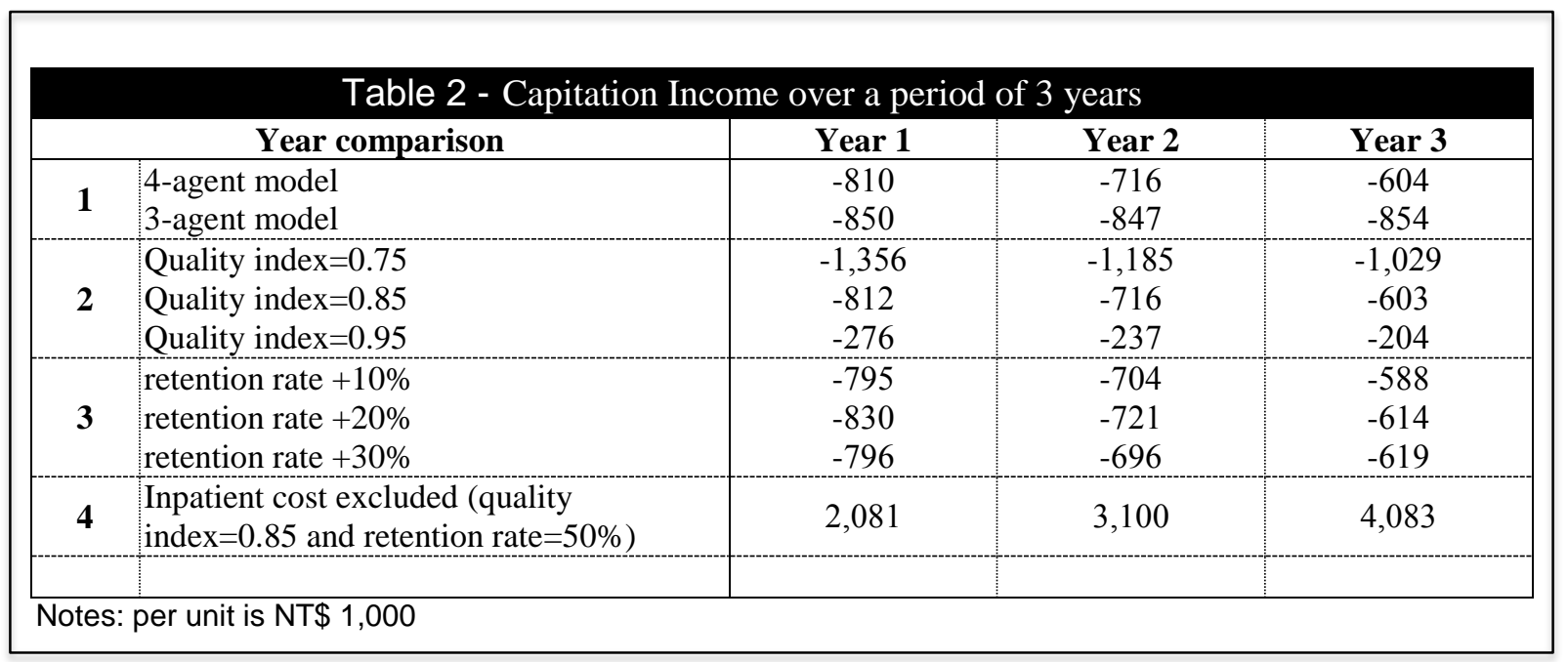

We proposed two system structures for comparison: one is the traditional triangular agent model, and the other is the $\mathrm{CHG}$ service model with four types of agents. The simulation environment was the same as with the benchmarked settings. The result is shown in Figure 7; the number of medical points claimed by the three-agent model is larger than that of the four-agent model over a period of three years. Conversely, the capitation income of the three-agent model is less than that of the four-agent model as shown in Table 2.

\section{Sensitivity Analysis: Quality Index}

Four indicators were used by the BNHI for establishing a quality index: diabetes control, community health promotion, degree of patient satisfaction, and primary care activities, such as Pap tests and influenza vaccinations. The fulfillment of these tasks was used to generate a quality index for calculating capitation rewards. We simulated the capitation system under three different quality indexes. The results shown in Table 3 indicate that financial loss is decreased if the quality index rate is increased, and in turn, the number of medical points is decreased. 


\begin{tabular}{|c|c|c|c|c|c|}
\hline \multicolumn{2}{|r|}{$\begin{array}{c}\text { Change rate } \\
\text { With interventions }\end{array}$} & Medical points & FFS income & $\begin{array}{c}\text { Capitation } \\
\text { income }\end{array}$ & Total income \\
\hline \multirow{2}{*}{1} & 4-agent model & $-2.03 \%$ & $-1.93 \%$ & $25.68 \%$ & $-1.64 \%$ \\
\hline & 3-agent model & $0.03 \%$ & $0.04 \%$ & $-0.38 \%$ & $0.04 \%$ \\
\hline \multirow{3}{*}{2} & Quality index $=0.75$ & $-1.52 \%$ & $-4.83 \%$ & $20.11 \%$ & $-4.54 \%$ \\
\hline & Quality index $=0.85$ & $-2.03 \%$ & $-1.93 \%$ & $25.68 \%$ & $-1.64 \%$ \\
\hline & Quality index $=0.95$ & $-2.08 \%$ & $-1.98 \%$ & $25.90 \%$ & $-1.89 \%$ \\
\hline \multirow{3}{*}{3} & retention rate $+10 \%$ & $-3.47 \%$ & $-2.57 \%$ & $42.93 \%$ & $-2.12 \%$ \\
\hline & retention rate $+20 \%$ & $-2.85 \%$ & $-2.54 \%$ & $30.60 \%$ & $-2.20 \%$ \\
\hline & retention rate $+30 \%$ & $-2.08 \%$ & $-1.80 \%$ & $27.42 \%$ & $-1.57 \%$ \\
\hline & $\begin{array}{l}\text { Inpatient cost was } \\
\text { excluded under the condition, } \\
\text { quality index }=0.85 \text { and } \\
\text { retention rate }=50 \%\end{array}$ & $-2.13 \%$ & $-1.99 \%$ & $96.18 \%$ & $0.95 \%$ \\
\hline
\end{tabular}

\section{Sensitivity Analysis: Patient Retention Rate}

According to medical payment history as provided by the Bureau of $\mathrm{NHI}$, the average retention rate of Zhishan-CHG patients was close to $50 \%$. As such, we set a retention rate of $50 \%$ as the benchmark rate for modeling. However, the retention rate might be changed by CHG's service strategies, such as an increase in the number of HLSE members, which may attract more people to access the Zhishan-CHG service system. Although the number of HLSE subscribers is randomly given in the system, if the number of subscribers is increased, the HLSE subscribers will become healthier via attending health promotion activities, which in turn will reduce medical payments accordingly. Due to tight engagement with
Zhishan-CHG, they may access ZhishanCHG services if they need to seek medical treatment as outpatients. We are interested in knowing the effects of different levels of retention rates on the financial outcomes of Zhishan-CHG through the simulation. The simulation results presented in Table 3 show that the financial loss of the capitation in the third year is decreased as the retention rate moves up to $90 \%$, and in Figure 8 , the results show that the total income decreases annually at three targeted ratios. 


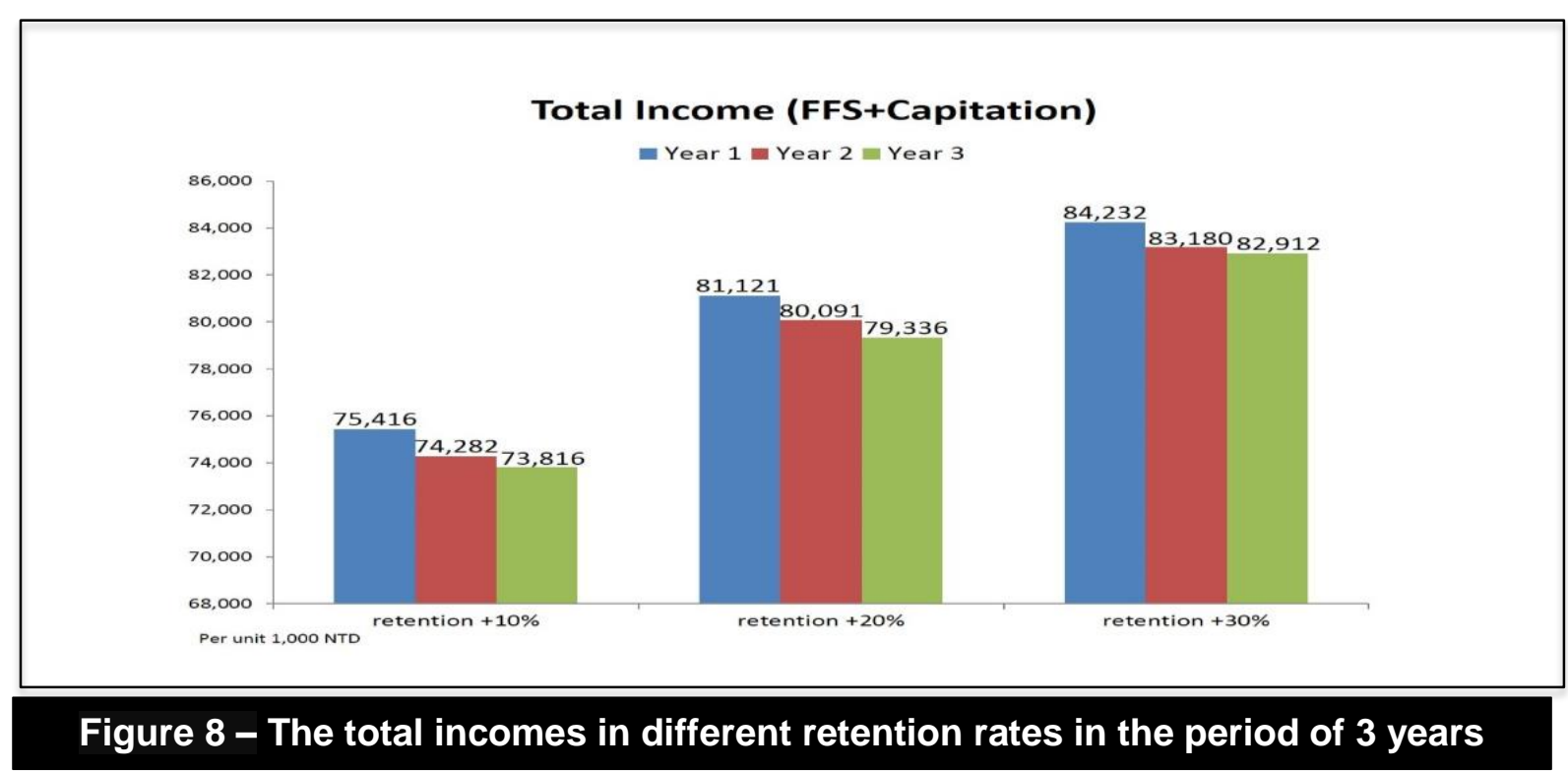

\section{The Change of Business Rule of Capitation}

Cox (2011) mentioned that for organizations to develop more efficient healthcare finance systems, they need to reject risk-assuming contracts, seek the elimination of inherently inefficient insurance risk transfers, and remain ever vigilant for risk transfers in occurring in any form, whatever the name used to describe them. In the previous four scenarios, the results showed that the current business rules set by $\mathrm{NHI}$ capitation policy brought financial losses to Zhishan$\mathrm{CHG}$ as shown in Table 3. Under current rules, the medical points given to each enrolled patient can be redeemed via inpatient or outpatient treatments. However, due to medical capacity limitations, ZhishanCHG only delivered outpatient medical services, and could not redeem medical points for its enrolled capitation patients if they needed to go for inpatient treatments in hospitals. Thus, although the retention rate and quality index moved to $80 \%$ and 0.95 respectively, Zhishan-CHG could not break even by the end of the third year, the end of the experimental project. Moreover, over $75 \%$ enrolled patients in Zhishan-CHG have chronic diseases, and these patients have a high probability of being hospitalized in the future, which creates financial risk for the CHG. Therefore, CHG capitation policy may not sustainably manage apparent system risks, even as the CHG has performed effectively to engage the majority of enrolled patients.

We conducted an experiment to estimate the financial outcomes if Zhishan-CHG did not bear the financial cost for inpatient treatments by excluding inpatient medical points from the total virtual points assigned to the enrolled patients. Under the benchmarked settings of the simulation system, the results shown in Table 3 indicate that the Zhishan-CHG could gain financial rewards from capitation payments to fill the gap in profits from the loss of FFS, and by such means reach financial balance. This implies that the system risk of the capitation policy for CHG-typed service systems could be better managed by proportionally subtracting inpatient costs from the financial risk borne by $\mathrm{CHGs}$.

\section{Conclusion}

The capitation payment scheme aimed to mend the financial gap created by the feefor-service payment scheme, which motivated medical institutes to provide more services than needed to generate more income (Cox, 2011). In this study, we 
evaluated the CHG-typed capitation service system using data from Zhishan-CHG, an experimental project sponsored by the $\mathrm{NHI}$, to evaluate the outcomes of the capitation policy. The multi-agent simulation system Swarm was adopted to simulate the stakeholders of the service system. We compared the traditional medical service system composed solely of the $\mathrm{NHI}$, medical institutes, and patients with an innovative service system which added a new agent, the health life social enterprise (HLSE), to form a service system with four types of agents. The results show a positive impact on reducing medical points, mitigating the financial burden of the NHI. We conducted sensitivity analyses for the variables of the quality index and retention rate, and the results show the effects of reducing ZhishanCHG's financial losses through increasing the quality of care and the retention rate.

However, the system risk created by inpatient treatment costs cannot be well managed given the current capitation policy. The exclusion of inpatient medical points from the total virtual points assigned to enrolled patients generates a positive financial outcome. Thus, this study suggests a potential capitation policy change for the $\mathrm{BNHI}$, which might co-create value with CHG-typed capitation services by proportionally subtracting inpatient costs from capitation reimbursements. This study has proved that financial incentives may be the key to change healthcare delivery. Moreover, the financial incentive may also enable organizational and cultural changes to sustain the healthcare service system (Kahn, 2009)

In summary, this research laid a foundation for evaluating new services, such as the capitation payment scheme. By using a multi-agent simulation system to predict possible outcomes under differently tuned conditions, decision-makers responsible for designing effective service systems can avoid costly real-world system changes while the outcomes of changes are still unclear. Using this foundation, we may model and simulate other types of healthcare service systems in capitation to shed light on the decisions pondered by $\mathrm{NHI}$ policymakers.

\section{References}

Baumeister, R. F., \& Leary, M. R. (1995). The need to belong: Desire for interpersonal attachments as a fundamental human motivation. Psychological Bulletin, 117(3), 497529.

Blackwell, B., Gutmann, M., \& Gutmann, L. (1988). Case review and quantity of outpatient care. American Journal of Psychiatry, 145(1003-1006), 1-1.

BNHI. (2011). National Health Insurance in Taiwan: 2011 Annual Report. Taipei, Taiwan: Bureau of National Health Insurance, Department of Health, Executive Yuan.

Cheng, T.-M. (2003). Taiwan's New National Health Insurance Program: Genesis And Experience So Far. Health Affairs, 22(3), 61-76.

Cheng, T.-M. (2009). Lessons From Taiwan's Universal National Health Insurance: A Conversation With Taiwan's Health Minister ChingChuan Yeh. Health Affairs, 28(4), 1035-1044.

Cleveland, W. (2012). Chapter 4: Capitation. In AMA (Ed.), Evaluating and Negotiating Emerging Payment Options (pp. 15). US: Practice management center of Amercian Medical Association (AMA).

Cox, T. (2011). Exposing the true risks of capitation financed healthcare. Journal of Healthcare Risk Management, 30(4), 34-41.

Fattal, O., Lampe, E., Barcelona, I., \& Muzina, D. (2005). Patient compliance with medication and follow-up appointments. Psychiatric Ann, 35, 165-178. 
Heirich, M. (1998). Rethinking health care: innovation and change in America: Westview Press.

Hiebeler, D. (1994). The swarm simulation system and individual-based modeling.

Hillman, A. L., Pauly, M. V., \& Kerstein, J. J. (1989). How do financial incentives affect physicians' clinical decisions and the financia

Hiebeler, D. (1994). The swarm simulation system and individual-based modeling.

Hillman, A. L., Pauly, M. V., \& Kerstein, J. J. (1989). How do financial incentives affect physicians' clinical decisions and the financial performance of health maintenance organizations? The New England Journal of Medicine, 321(2), 86.

Chan, W. S. H.(2010). Taiwan's healthcare report 2010. The EPMA Journal, 1(4), 563-585.

Hubley, J., \& Copeman, J. (2008). Chapter 6 Health Promotion with Groups Practical health promotion. UK: Polity.

Kahn, C. N. (2009). Payment reform alone will not transform health care delivery. Health Affairs, 28(2), w216-w218.

Lin, F.-r., \& Pai, Y.-H. (2000). Using multiagent simulation and learning to design new business processes. Systems, Man and Cybernetics, Part A: Systems and Humans, IEEE Transactions on, 30(3), 380-384.

Lin, F.-R., Sung, Y.-W., \& Lo, Y.-P. (2003). Effects of trust mechanisms on supply-chain performance: A multiagent simulation study. International Journal of Electronic Commerce, 9(4), 9-112.

Lin, F.-R., Tan, G. W., \& Shaw, M. J. (1999). Multiagent enterprise modeling. Journal of Organizational Computing and Electronic Commerce, 9(1), 7-32.
Lusch, R. F., Vargo, S. L., \& O'Brien, M. (2007). Competing through service: Insights from service-dominant logic. Journal of Retailing, 83(1), 5-18.

McColl-Kennedy, J. R., Vargo, S. L., Dagger, T. S., Sweeney, J. C., \& van Kasteren, Y. (2012). Health Care Customer Value Cocreation Practice Styles. Journal of Service Research.

McLeroy, K. R., Bibeau, D., Steckler, A., \& Glanz, K. (1988). An ecological perspective on health promotion

programs. Health Educ Q, 15(4), 351-377.

Miller, H. D. (2009). From volume to value: better ways to pay for health care. Health Affairs, 28(5), 1418-1428.

Minar, N., Burkhart, R., Langton, C., \& Askenazi, M. (1996). The swarm simulation system: A toolkit for building multi-agent simulations. Paper presented at the Working Paper 96-06-042, Santa Fe Institute, Santa Fe.

Moreno, A. (2003). Medical applications of multi-agent systems. Computer Science and Mathematics Department, University of Rovira, Spain.

Moreno, A., Isern, D., \& Sánchez, D. (2003). Provision of agent-based health care services. Al Communications, 16(3), 167.

Nealon, J. (2013). Agentcities healthcare working group. Retrieved Feb. 4th, 2013, from http://www.agentcities.org/

Nealon, J., \& Moreno, A. (2003). Agentbased applications in health care. in $\mathrm{J}$. Nealon and A. Moreno (eds.), Applications of Software Agent Technology in the Health Care Domain, (pp.3-18) Basel Switzerland: Birkhauser.

Neuwelt, P. M. (2012). Community participation in primary care: what 
dsoes it mean 'in practice'? Joural of Primary Health Care, 4(1), 30-38.

Porter, M. E. (2009). A Strategy for Health Care Reform - Toward a ValueBased System. New England Journal of Medicine, 361(2), 109-112.

Porter, M. E., \& Teisberg, E. O. (2006). Redefining Health Care: Creating Value-Based Competition on Results. Boston, Mass.: Harvard Business School Press.

Rice, N., \& Smith, P. (1999). Approaches to capitation and risk adjustment in health care: an international survey. UK:: Centre for Health Economics, University of York.

Roberge, D., Beaulieu, M.-D., Haddad, S., Lebeau, R., \& Pineault, R. (2001). Loyalty to the regular care provider: patients' and physicians' views. Family Practice, 18(1), 53-59.

Roberts-DeGennaro, M. (1987). Patterns of exchange relationships in building a coalition. Administration in Social Work, 11(1), 59-67.

Sterman, J. D. (2006). Learning from evidence in a complex world. American Journal of Public Health, 96(3), 505-514.

van der Hoek, W., \& Wooldridge, M. (2008). Multi-agent systems. Foundations of Artificial Intelligence, 3, 887-928.

Vargo, S. L., \& Lusch, R. F. (2008). Servicedominant logic: continuing the evolution. Journal of the Academy of Marketing Science, 36(1), 1-10.

Vargo, S. L., Maglio, P. P., \& Akaka, M. A. (2008). On value and value cocreation: A service systems and service logic perspective. European Management Journal, 26(3), 145-152.

WHO. (2010). The world health report: health systems financing: the path to universal coverage (M. Reid Ed.). Switzerland: WHO Press, World Health Organization.
Wooldridge, M., \& Jennings, N. R. (1995). Intelligent agents: Theory and practice. Knowledge engineering review, 10(2), 115-152.

Xiang, Y. (2002). Probabilistic Reasoning in Multiagent Systems: A graphical models approach: Cambridge University Press.

Yu, Y.-C. (2007). The future clinics in the perspective of chronical disease care model (3)-- the reform of healthcare organizations. Journal of Healthcare Quality, 1(5), 75-83. (in Chinese) 